\title{
Neuroimaging in the Emergency Department for Patients with Symptoms of Vitreoretinal Traction
}

\author{
Adnan Malick, MD ${ }^{1,2}$ Peter J. Belin, MD ${ }^{1}$ Vincent Deramo, MD $^{1,3} \quad$ Jules Winokur, MD ${ }^{1}$ \\ Matthew Gorski, MD ${ }^{1}$ Dorjan Pantic, MD ${ }^{4}$ Evan R. Schloss, MD ${ }^{5}$ David Fastenberg, MD ${ }^{1,3}$
}

1 Department of Ophthalmology, Donald and Barbara Zucker School of Medicine at Hofstra/Northwell, Great Neck, New York

${ }^{2}$ Department of Ophthalmology, The Ohio State University Wexner

Medical Center, Havener Eye Institute, Columbus, Ohio Ophthalmology, Donald and Barbara Zucker School of Medicine at Hofstra/Northwell, 600 Northern Boulevard, Suite 214, Great Neck,

${ }^{3}$ Long Island Vitreoretinal Consultants, Great Neck, New York

${ }^{4}$ Department of Neurology, Donald and Barbara Zucker School of Medicine at Hofstra/Northwell, Manhasset, New York

${ }^{5}$ Department of Emergency Medicine, Donald and Barbara Zucker

School of Medicine at Hofstra/Northwell, Manhasset, New York NY 11021 (e-mail: petebelin@gmail.com).

J Acad Ophthalmol 2019;11:e18-e23.

\begin{abstract}
Keywords

- diagnostic imaging

- visual complaints

- management

- ophthalmology

Eye conditions result in 2.4 million emergency department (ED) visits annually. The purpose of this study is to investigate the management of patients with symptoms of vitreoretinal traction. Retrospective observational study was performed in three Northwell Health EDs between January 2014 and January 2017. Patients with monocular flashes, floaters, or changes in visual field for whom ophthalmology were consulted. Ninety-six patients were included ( 45 female), mean age 58.4 years. Complaints included floaters in 47 (49\%), visual field changes in 39 (41\%), and flashes in $28(29 \%)$. Eighteen patients (19\%) presented with more than one symptom. Of 24 patients with documented eye examinations by emergency physicians, 10 included confrontational visual fields (CVF). Before ophthalmology consultation, tests included blood work in 29 patients (30\%), computerized axial tomography (CT) head in 33 (34\%), orbit ultrasound in two (2\%), magnetic resonance imaging (MRI) head in 1 one (1\%), and erythrocyte sedimentation rate (ESR)/C-reactive protein (CRP) in one (1\%). Diagnoses by ophthalmologists were posterior vitreous detachment in 44 patients (46\%), vitreous hemorrhage in 31 (32\%), retinal detachment or tears in $12(13 \%)$, central retinal artery occlusion in six (6\%), and central or branch retinal vein occlusions in three (3\%). Of 12 patients with retinal detachments or tears, 5 (42\%) underwent surgery within 1 week. In patients presenting to the ED with symptoms of vitreoretinal traction, over $30 \%$ underwent imaging and blood work before ophthalmology consultation. Signs and symptoms of retinal pathology should be recognized promptly. Triaging, focused examination, and a detailed history may lead to fewer imaging studies and more cost savings.
\end{abstract}

Eye conditions are responsible for 2.4 million emergency department (ED) visits per year. ${ }^{1}$ Previous studies have investigated common ophthalmic diagnoses and management in patients seeking care in the ED. ${ }^{2-7}$ Though a large percentage

(1D) Dr. Peter J. Belin's ORCID is https://orcid.org/0000-0002-1554-
5850.

received

February 18, 2019

accepted after revision

June 24, 2019
DOI https://doi.org/

10.1055/s-0039-1694037. ISSN 2475-4757. of visits are due to common diseases such as conjunctivitis, corneal abrasions, dry eyes, and blepharitis, there are many situations that require urgent ophthalmic care or intervention. As a result, ophthalmologists are routinely consulted for a variety of complaints. Among these diagnoses, studies have documented between 7.6 and $19.4 \%$ of consultations called due to retinal disorders. ${ }^{2,5,7}$

Copyright $\odot 2019$ by Thieme Medical Publishers, Inc., 333 Seventh Avenue, New York, NY 10001, USA. Tel: +1(212) 584-4662.
License terms

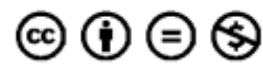


Prior to consultation by an ophthalmologist, patients are typically triaged by ED staff that examine patients and order tests they deem necessary. ED physicians also determine which patients require examination by an ophthalmologist. Though most retinal pathology can be identified through a dilated examination of the fundus or with B-scan ultrasonography, definitively diagnosing specific conditions may be challenging based on symptoms alone. While subjective complaints of reduced visual acuity, visual field changes, floaters, and photopsias may result from retinal disorders, other etiologies may cause similar symptoms.

Monocular flashes of light are commonly caused by vitreoretinal traction, which may be associated with retinal breaks, retinal detachment (RD), or posterior vitreous detachment (PVD). Visual field changes are also a common presenting symptom for retinal disorders or optic nerve disease. Similar symptoms may occur in patients with central nervous system (CNS) disorders, such as migraine, ischemic stroke, intracranial hemorrhage, occipital seizure, vertebrobasilar insufficiency, and visual hallucinations, although these are frequently binocular. $^{8}$

As reduced vision, floaters, and several other symptoms could be a result of retinal disorders, neurological disorders, or other ocular and systemic conditions, a thorough ocular history and examination by ED staff is vital. Anterior segment examination including pupils and confrontational visual fields (CVF), identification of laterality, and a thorough history of symptom onset and associated symptoms are helpful in narrowing the differential diagnosis and guiding management.

The main purpose of this study is to investigate ED management of patients presenting with monocular visual complaints. We differentiated a subset of patients with symptoms correlating with retinal disease. While previous studies report the epidemiology of ophthalmology consultations in EDs, there are no studies to our knowledge investigating ED management of patients prior to ophthalmology consultation for symptoms of vitreoretinal traction.

\section{Materials and Methods}

The Institutional Review Board of Northwell Health approved this study. We performed a retrospective chart review of all consecutive patients evaluated by four physicians on the ophthalmology consult service among three hospitals in the Northwell Health system between January 1, 2014 and December 31, 2016. In these hospitals, an ophthalmologist is available on call for 24 hours a day, 365 days a year. The consulting ophthalmologist is typically able to see every patient within 30 to 60 minutes from when the consult is placed. Inclusion criteria for the study were patients complaining of monocular flashes, floaters, or changes in visual field, without systemic neurological symptoms.

All patients presented to the emergency department and were evaluated and worked up by the ED staff, then subsequently by the ophthalmology department. Patient demographics, past medical and ocular history, subjective complaints, emergency physician examinations, testing ordered prior to ophthalmology consultation, and ophthal-

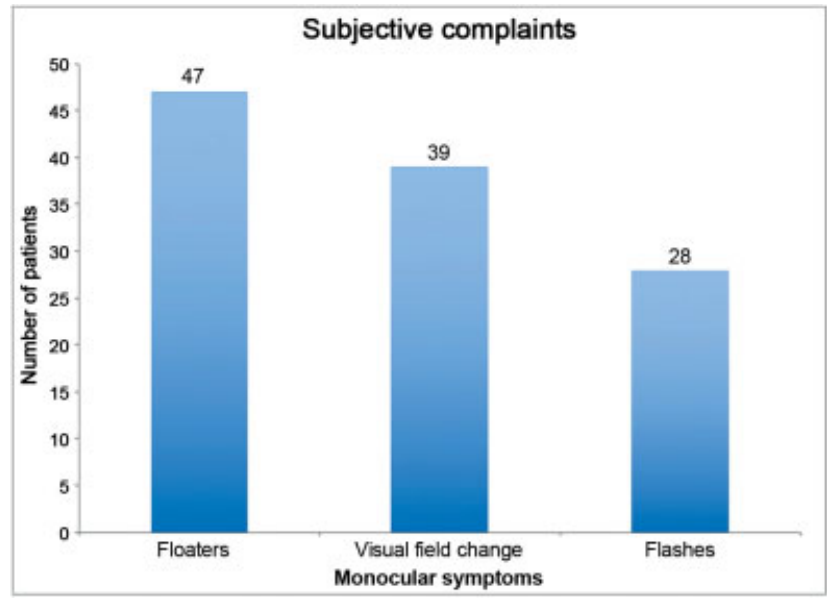

Fig. 1 Subjective complaints reported by patients.

mology examination results were documented. Outpatient photocoagulation and surgical procedures were also reviewed for patients requiring intervention by retinal specialists. We obtained data using electronic medical records and paper charts. Patients with binocular symptoms and patients with additional systemic symptoms requiring stroke workup were excluded.

\section{Results}

A total of 100 patients were identified. Four of them were excluded since full ophthalmic examination was not recorded. Ninety-six patients were included and comprised this study with 45 females (47\%) and 51 males (53\%). The mean age of patients was 58.4 years. Subjective complaints included floaters in 47 patients (49\%), changes in visual field in 39 patients (41\%), and flashes in 28 patients (29\%) (-Fig. 1). Eighteen patients (19\%) presented with more than one symptom.

Among the 24 patients who had documented eye examinations by emergency physicians, 10 included confrontational visual field examinations. Prior to consultation by an ophthalmologist, tests ordered by the ED included routine blood work (complete blood count [CBC] and basic metabolic panel [BMP])in 29 patients (30\%), computerized axial tomography (CT) of the head (with or without orbits) in 33 patients (34\%), ultrasound of orbit by the ED physician in two patients (2\%), magnetic resonance imaging (MRI head) in one patient (1\%), and serum erythrocyte sedimentation rate (ESR)/C-reactive protein (CRP) in one patient (1\%; - Fig. 2).

Final diagnoses of patients after examination by an ophthalmologist were found to be posterior vitreous detachment in 44 patients (46\%), vitreous hemorrhage in 31 patients (32\%), retinal detachment in 11 patients (11\%), central retinal artery occlusion in six patients (6\%), central or branch retinal vein occlusions in three patients (3\%), and retinal tear in one patient (1\%; - Fig. 3 ).

Of the 12 patients with retinal detachments or tears (11 with retinal detachments and one with a tear), 4 underwent imaging studies in the ED (33\%). All imaging studies were 


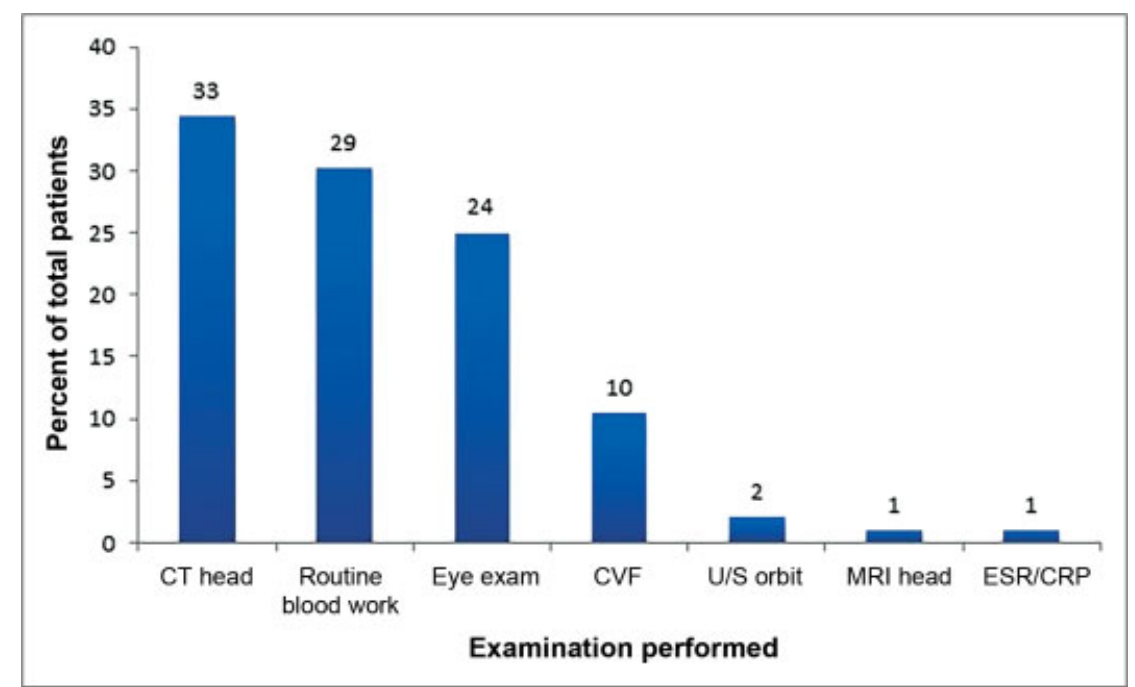

Fig. 2 CRP, C-reactive protein; CT, computed tomography; CVF, confrontational visual fields; ESR, erythrocyte sedimentation rate; MRI, magnetic resonance imaging; $\mathrm{U} / \mathrm{S}$, ultrasound.

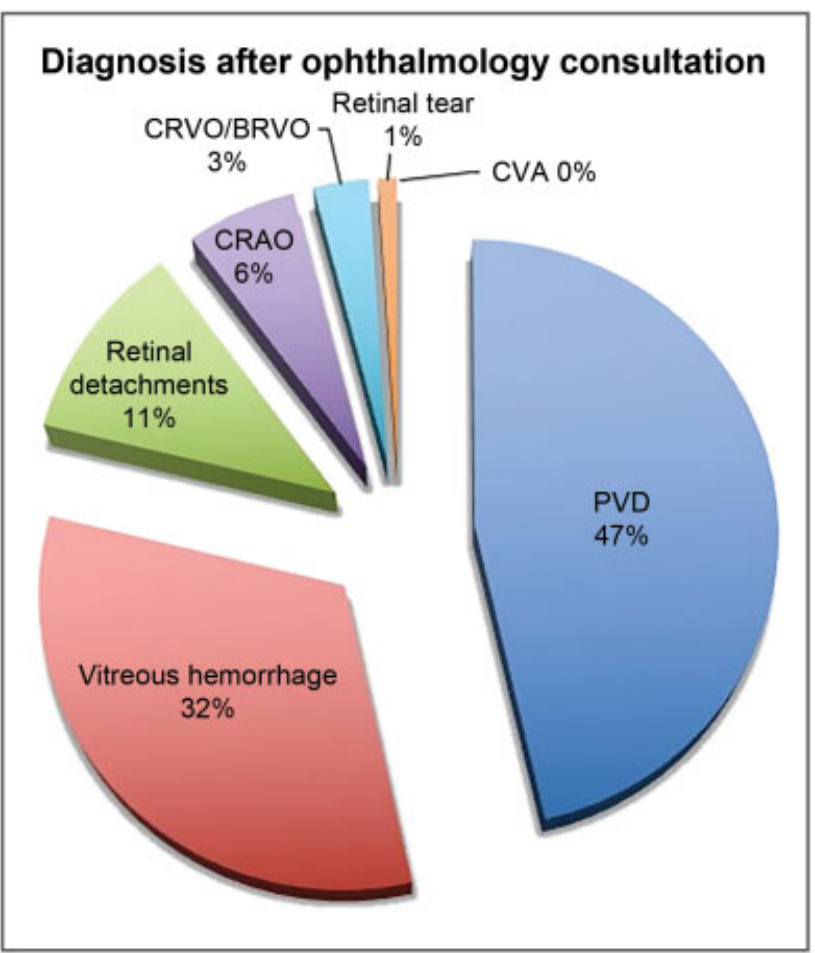

Fig. 3 BRVO, branch retinal vein occlusion; CRAO, central retinal artery occlusion; CRVO, central retinal vein occlusion; CVA, cerebrovascular accident; PVD, posterior vitreous detachment.

performed prior to the dilated fundus examination by an ophthalmologist. No patients underwent further imaging studies (aside from B-scan ultrasonography) after diagnosis of retinal pathology by an ophthalmologist.

Of the 12 patients with retinal detachments or tears, five patients (42\%) underwent surgical repair within 1 week of examination, ranging from same day to 6 days after presentation. Information was not available for six patients who continued their care elsewhere. The one patient found to have a retinal tear received barrier laser photocoagulation on the same day of the ED visit.

\section{Discussion}

For any clinician, the challenge of evaluating and managing patients with ocular symptoms lies in correctly identifying the etiology of the complaint. As the differential for symptoms of flashes, floaters, and visual field changes is broad, many physicians utilize imaging as a first-line modality to rule out life-threatening causes. In our study, over $30 \%$ of patients with monocular symptoms indicative of retinal pathology underwent either CT or MRI scan. With a mean age of over 58 years and a history of hypertension in $48 \%$ of patients included in our study, it is possible that ordering of imaging may be related to the concern for stroke. Increased availability of CT scans in most ED settings may also be playing a role in its increased use.

\section{Identification of Laterality}

As with any patient who presents to the ED or outpatient office, a careful history and physical examination is imperative. When narrowing the differential in patients with ocular complaints, a key aspect to identify is laterality. Although exceptions exist, patients presenting with acute monocular visual field changes, flashes, or floaters rarely experience such symptoms due to neurological disease. Another aspect of the history to consider is whether the symptoms are "positive" such as lights or colors, or "negative" such as darkened vision, with negative visual phenomena more concerning for intracranial pathology. Flashes of light may precede migraine headaches or occipital seizures, but they typically occur bilaterally and are episodic. Patients with idiopathic intracranial hypertension (IIH) may have binocular transient visual obscurations. Similarly, field loss caused by stroke or other disorders of the CNS is typically bilateral and homonymous, due to crossing of nasal fibers at the optic chiasm. Few exceptions exist, such as a monocular temporal crescent deficit associated with anterior occipital infarcts. Any concern from the history for CNS disease warrants a comprehensive neurological examination. Focal lateralizing 
deficits on the neurological examination such as facial droop, ataxia, hemiparesis, or hemisensory loss in the setting of a visual field cut may warrant neuroimaging.

In our study, 10 of 96 patients had a documented CVF performed by the ED. Of the 10 patients with confrontational visual field examinations in the ED, only 1 patient was sent for CT scan. The one patient who was sent for imaging was thought to have a bitemporal visual field deficit by the ED (which was not found by the ophthalmologist on examination). The other nine patients were found to have either full CVF's or monocular deficits, and no neuroimaging studies were ordered by the staff. As all of the patients in our study presented with monocular symptoms, CVF examination and identification of laterality alone reduced the incidence of imaging significantly. Although the sample size of patients having CVF examinations in the ED is too small to achieve statistical significance, we believe a more thorough examination by the ED alone or consultation by an ophthalmologist may decrease neuroimaging studies.

Our study demonstrated that CT or MRI of the head did not contribute to the diagnosis of retinal disease in patients with symptoms of monocular flashes, floaters, or visual field changes presenting to the emergency department. In fact, neither the American Academy of Ophthalmology nor the Wills Eye Manual recommends CT or MRI in the workup for retinal detachments, retinal tears, or PVDs. ${ }^{8}$ Although carotid ultrasound and certain blood work is recommended for CRAOs (central retinal artery occlusion) and CRVOs (central retinal vein occlusion), a CT scan or MRI of the head is not part of the workup for either. ${ }^{8}$ Evaluation by an ophthalmologist which includes dilated examination of the fundus or B-scan ultrasonography is the definitive means to identify diagnoses such as retinal detachments, posterior vitreous detachments, vitreous hemorrhages, and retinal holes or tears.

\section{CT Scan and MRI}

When used in the appropriate setting, a CT scan or MRI is fundamental in diagnosing certain pathologies. Prompt diagnosis of cerebral ischemia, hemorrhage, aneurysms, and intracranial/orbital masses is crucial, and may aid in guiding time-sensitive therapy. When employed liberally, imaging studies may lead to increased health care costs and extended hospital stays in situations where they are not necessarily indicated. A review of national data from 1996 through 2007 reveals a sharp increase in the use of CT scans in the emergency department over the past few years. According to findings from the University of Michigan Health System, just 3.2\% of ED patients received CT scans in 1996, while $13.9 \%$ of patients evaluated in 2007 received them, correlating to a $330 \%$ increase in just 12 years. ${ }^{9}$

Though the cost of a CT scan of the head varies among health systems, several studies cite costs of $\$ 1,000$ or more. ${ }^{10-13}$ Further workup of incidental findings could lead to potentially greater expenditures and time spent in the hospital setting. With such a stark increase in the use of imaging, several studies have analyzed the utility and effectiveness of obtaining scans in certain situations. As the emphasis on cost-effective care intensifies in our society, there must be standards and guide- lines to appropriate proper care. Studies argue a low-diagnostic value in the use of CT scans in several situations, including certain cranial nerve palsies, ${ }^{11}$ vertigo, ${ }^{12}$ and minor head trauma. ${ }^{13}$ All authors place emphasis on attaining a thorough patient history and a good physical and neurological examination. A similar, if not stronger argument can be made for our subset of patients.

In fact, according to the Centers for Medicare and Medicaid Services (CMS) 2019 physician fee schedule, the global reimbursement rate in the NYC suburbs/Long Island locality (where this study took place) for a CT head without contrast is $\$ 138.59$, MRI brain without contrast is $\$ 271.38$, CBC is $\$ 7.18$, BMP is $\$ 11.74$, ESR is $\$ 4.27$, and CRP is $\$ 5.75$. In our study, these costs would include $\$ 548.68$ for routine blood work in 29 patients, $\$ 4,573.47$ for 33 CT head without contrast in 33 patients, $\$ 271.38$ for MRI brain without contrast in one patient, and $\$ 10.02$ for ESR/CRP in one patient for a total of $\$ 5,403.55$ in unnecessary testing. Reimbursement from commercial insurance can vary but is typically higher than the prices set by CMS. Therefore, the actual cost could be much higher than this conservative estimate based on the CMS physician fee schedule.

As mentioned previously, there is minimal yield and substantial cost obtaining neuroimaging in patients with RDs, PVDs, retinal tears/holes, or vitreous hemorrhages. A meticulous history and physical examination can be done quickly, reduce delay in diagnosis, reduce cost of care, and improve patient satisfaction.

Furthermore, awareness of potential radiation hazards associated with radiological examinations is crucial for the risk-benefit evaluation of attaining diagnostic imaging. Mean doses of radiation from a simple CT head could range between 2 and 2.9 millisieverts (mSv). ${ }^{14,15}$ Though the exact magnitude of risk is controversial at lower doses, the cumulative dose of multiple studies may potentially expose patients to the side effects of radiation.

One possible factor determining the diagnostic algorithm may be the availability of ophthalmologists for timely consultation. If ophthalmologists are not readily available, CT scans may be ordered more frequently and vice versa. However, this reason would not be a contributing factor for the present study as the Northwell system has 24/7 ophthalmology coverage.

\section{Length of Stay}

Along with increasing health care costs and the hazards associated with imaging, another facet to consider is delay in diagnosis. While a CT scan only takes a few minutes, the time associated with ordering, performing, and interpreting a scan may take hours. Shift changes in the ED may also occur in the interim, adding to the delay in diagnosis. All the 33 patients (100\%) who underwent imaging in our study, all scans were ordered prior to ophthalmology consultation. This delay in diagnosis may potentially lengthen hospital stay and postpone definitive management of certain time-sensitive conditions.

\section{Management of Acute Retinal Pathology}

Patients with symptomatic retinal tears from vitreoretinal traction are at significant risk for developing rhegmatogenous 
retinal detachment (RRD). Untreated symptomatic horseshoe tears have been reported to cause retinal detachment in up to $55 \%$ of cases. ${ }^{16}$ When treated appropriately, the risk of RRD falls to 1.4 to $7.8 \%{ }^{16}$ Most sources, including the American Academy of Ophthalmology, agree that retinal breaks with persistent vitreous traction should be treated with prompt laser retinopexy. ${ }^{17}$ Though only one patient in our sample size of 96 was diagnosed with a retinal tear, this patient was referred to a retina specialist for laser retinopexy. As the equipment to treat acute retinal tears is not typically available in every emergency department, and the diagnostic equipment the office setting may surpass that found in the ED, prompt referral to an ophthalmologist is ideal.

Similar implications exist for patients with retinal detachments. Though retinal detachments are not necessarily considered as surgical emergencies, a degree of time sensitivity exists. Hassan et al investigated the effect of duration of repair on final visual acuity for macula-off retinal detachments. Results showed visual acuity of 20/ 40 or better in $71 \%$ of eyes with repair within 10 days or less, $27 \%$ of eyes with repair 11 days to 6 weeks, and $14 \%$ of eyes with repair after 6 weeks. ${ }^{18}$ Similarly, Burton has shown that operations within 9 days had a better chance of achieving visual acuity $>20 / 50 .{ }^{19}$ Another recent study demonstrated that starting 6 days following development of macula-off retinal detachment, the visual outcome could be expected to worsen progressively. ${ }^{20}$

As involvement of the fovea is the greatest predictor of visual and anatomical outcomes in patients with RD, surgery is pursued more urgently in patients in whom the macula is attached. Though some sources suggest equivalent visual outcomes up to 3 to 5 days after detachment, ${ }^{21-23}$ most clinicians choose to repair these detachments promptly. Factors including timing of symptom onset and proximity to the fovea can better help guide management on a case-by-case basis. ${ }^{24,25}$ Though not every patient presenting to the ED with flashes and floaters necessarily needs a same-day consultation by ophthalmology, symptom identification can be used to triage care appropriately and help better prepare patients and surgeons for potential intervention. Ophthalmology consultation may be helpful to determine if additional testing, like CT/MRI is needed in the setting of a normal neurological and systemic examination. $^{24,25}$

\section{Limitations}

Our study has several limitations. One, our exclusion criteria introduces a bias whereby patients who may have presented with monocular flashes, floaters, or visual field changes and systemic symptoms were excluded. Second, there was significant selection bias, given that ophthalmology was consulted quite downstream in the workup of these patients. For example, ophthalmology consults would not be called on patients who had positive CT imaging in the ED. Similarly, in the prehospital setting patients could present to outpatient ophthalmologists or neurologists and be sent to the ED with a known neurologic diagnosis, so an ED ophthalmology consult would never be called. Therefore, the patients included in this study were already biased toward having a nonneurologic diagnosis.

\section{Conclusions}

In this retrospective study, patients presenting with monocular flashes, floaters, or visual field changes, over 30\% underwent imaging studies including CT scans and MRI's. Signs and symptoms of retinal pathology should be recognized promptly so that consultation with or referral to an ophthalmologist can be made. Likewise, a focused history and neurologic examination should be obtained to diagnose CNS mimickers of monocular disease. Confrontation visual field testing in the ED setting may be helpful in identifying monocular versus binocular visual field loss. Appropriate triaging and management can lead to more targeted diagnosis, fewer imaging studies, higher cost savings, and reduced exposure to radiation. Future multicenter prospective studies should evaluate all patients who present to the ED with monocular flashes, floaters, or visual field changes regardless of systemic or neurologic signs, while strictly excluding patients sent to the ED with a known neurologic diagnosis, to minimize selection bias and more faithfully determine the role of neuroimaging.

\section{Funding \\ None.}

Conflict of Interest

None declared.

\section{References}

1 Hing E, Hall MJ, Ashman JJ, Xu J. National hospital ambulatory medical care survey: 2007 outpatient department summary. Natl Health Stat Rep 2010;28(28):1-32

2 Kumar NL, Black D, McClellan K. Daytime presentations to a metropolitan ophthalmic emergency department. Clin Exp Ophthalmol 2005;33(06):586-592

3 Jan S, Khan S, Khan MN, Iqbal A, Mohammad S. Ocular emergencies. J Coll Physicians Surg Pak 2004;14(06):333-336

4 Voon LW, See J, Wong TY. The epidemiology of ocular trauma in Singapore: perspective from the emergency service of a large tertiary hospital. Eye (Lond) 2001;15(Pt 1):75-81

5 Edwards RS. Ophthalmic emergencies in a district general hospital casualty department. Br J Ophthalmol 1987;71(12):938-942

6 Vernon SA. Analysis of all new cases seen in a busy regional centre ophthalmic casualty department during 24 -week period. J R Soc Med 1983;76(04):279-282

7 Rizzuti AE, Vastardi M, Hajee M, Lazzaro DR. Scope of resident ophthalmology consultation service and patient follow-up rates at a level 1 trauma center in Brooklyn, New York. Clin Ophthalmol 2013;7:643-647

8 Khare GD, Symons RC, Do DV. Common ophthalmic emergencies. Int J Clin Pract 2008;62(11):1776-1784

9 Kocher KE, Meurer WJ, Fazel R, Scott PA, Krumholz HM, Nallamothu BK. National trends in use of computed tomography in the emergency department. Ann Emerg Med 2011;58(05):452-62.e3

10 Ahsan SF, Syamal MN, Yaremchuk K, Peterson E, Seidman M. The costs and utility of imaging in evaluating dizzy patients in the emergency room. Laryngoscope 2013;123(09):2250-2253

11 Lee AG, Hayman LA, Brazis PW. The evaluation of isolated third nerve palsy revisited: an update on the evolving role of magnetic 
resonance, computed tomography, and catheter angiography. Surv Ophthalmol 2002;47(02):137-157

12 Kerber KA. Vertigo and dizziness in the emergency department. Emerg Med Clin North Am 2009;27(01):39-50, viii

13 Miller EC, Holmes JF, Derlet RW. Utilizing clinical factors to reduce head CT scan ordering for minor head trauma patients. J Emerg Med 1997;15(04):453-457

14 You JS, Lee HJ, Chung YE, et al. Diagnostic radiation exposure of injury patients in the emergency department: a cross-sectional large scaled study. PLoS One 2013;8(12):e84870

15 Worrall JC, Jama S, Stiell IG. Radiation doses to emergency department patients undergoing computed tomography. CJEM 2014;16(06):477-484

16 Jalali S. Retinal detachment. Community Eye Health 2003;16(46): 25-26

17 Silva RA, Blumenkranz MS. Prophylaxis for Retinal Detachments. Available at: https://www.aao.org/munnerlyn-laser-surgery-center/prophylaxis-retinal-detachments. Accessed July 10, 2019

18 Hassan TS, Sarrafizadeh R, Ruby AJ, Garretson BR, Kuczynski B, Williams GA. The effect of duration of macular detachment on results after the scleral buckle repair of primary, macula-off retinal detachments. Ophthalmology 2002;109(01):146-152
19 Burton TC. Recovery of visual acuity after retinal detachment involving the macula. Trans Am Ophthalmol Soc 1982;80:475-497

20 Diederen RM, La Heij EC, Kessels AG, Goezinne F, Liem AT, Hendrikse F. Scleral buckling surgery after macula-off retinal detachment: worse visual outcome after more than 6 days. Ophthalmology 2007;114(04):705-709

21 Ho SF, Fitt A, Frimpong-Ansah K, Benson MT. The management of primary rhegmatogenous retinal detachment not involving the fovea. Eye (Lond) 2006;20(09):1049-1053

22 Ehrlich R, Niederer RL, Ahmad N, Polkinghorne P. Timing of acute macula-on rhegmatogenous retinal detachment repair. Retina 2013;33(01):105-110

23 Gorovoy IR, Porco TC, Bhisitkul RB, de Juan E Jr., Schwartz DM, Stewart JM. Same-day versus next-day repair of fovea-threatening primary rhegmatogenous retinal detachments. Semin Ophthalmol 2016;31(03):219-225

24 Ross WH, Kozy DW. Visual recovery in macula-off rhegmatogenous retinal detachments. Ophthalmology 1998;105(11):2149-2153

25 van Bussel EM, van der Valk R, Bijlsma WR, La Heij EC. Impact of duration of macula-off retinal detachment on visual outcome: a systematic review and meta-analysis of literature. Retina 2014;34 (10):1917-1925 